\title{
The PALM-3000 high-order adaptive optics system for Palomar Observatory
}

\section{Antonin H. Bouchez, Richard G. Dekany, John R. Angione, Christoph Baranec, Matthew C. Britton, et al.}

Antonin H. Bouchez, Richard G. Dekany, John R. Angione, Christoph Baranec, Matthew C. Britton, Khanh Bui, Rick S. Burruss, John L. Cromer, Stephen R. Guiwits, John R. Henning, Jeff Hickey, Daniel L. McKenna, Anna M. Moore, Jennifer E. Roberts, Thang Q. Trinh, Mitchell Troy, Tuan N. Truong, Viswa Velur, "The PALM-3000 high-order adaptive optics system for Palomar Observatory," Proc. SPIE 7015, Adaptive Optics Systems, $70150 Z$ (10 July 2008); doi: 10.1117/12.789777

SPIE Event: SPIE Astronomical Telescopes + Instrumentation, 2008, Marseille, France 


\title{
The PALM-3000 high-order adaptive optics system for Palomar Observatory
}

\author{
Antonin H. Bouchez ${ }^{*} a$, Richard G. Dekany ${ }^{a}$, John R. Angione ${ }^{b}$, Christoph Baranec ${ }^{a}$, \\ Matthew C. Britton ${ }^{a}$, Khanh Bui ${ }^{a}$, Rick S. Burruss ${ }^{b}$, John L. Cromer ${ }^{a}$, Stephen R. Guiwits ${ }^{b}$, \\ John R. Henning ${ }^{c}$, Jeff Hickey ${ }^{c}$, Daniel L. McKenna ${ }^{c}$, Anna M. Moore ${ }^{a}$, Jennifer E. Roberts ${ }^{b}$, \\ Thang Q. Trinh ${ }^{b}$, Mitchell Troy ${ }^{b}$, Tuan N. Truong ${ }^{b}$, Viswa Velur ${ }^{a}$ \\ ${ }^{a}$ Caltech Optical Observatories, California Institute of Technology, Pasadena, CA, USA 91125; \\ ${ }^{b}$ Jet Propulsion Laboratory, California Institute of Technology, Pasadena, CA, USA 91109; \\ ${ }^{c}$ Palomar Observatory, California Institute of Technology, Palomar Mountain, CA, USA 92060
}

\begin{abstract}
Deployed as a multi-user shared facility on the 5.1 meter Hale Telescope at Palomar Observatory, the PALM-3000 highorder upgrade to the successful Palomar Adaptive Optics System will deliver extreme AO correction in the near-infrared, and diffraction-limited images down to visible wavelengths, using both natural and sodium laser guide stars. Wavefront control will be provided by two deformable mirrors, a 3368 active actuator woofer and 349 active actuator tweeter, controlled at up to $3 \mathrm{kHz}$ using an innovative wavefront processor based on a cluster of 17 graphics processing units. A Shack-Hartmann wavefront sensor with selectable pupil sampling will provide high-order wavefront sensing, while an infrared tip/tilt sensor and visible truth wavefront sensor will provide low-order LGS control. Four back-end instruments are planned at first light: the PHARO near-infrared camera/spectrograph, the SWIFT visible light integral field spectrograph, Project 1640, a near-infrared coronagraphic integral field spectrograph, and 888Cam, a high-resolution visible light imager.
\end{abstract}

Keywords: Adaptive Optics, Extreme Adaptive Optics, Laser Guide Stars

\section{INTRODUCTION}

PALM-3000 is a high-precision AO upgrade to the successful PALMAO adaptive optics system ${ }^{1}$ on the 5.1 meter Hale Telescope at Palomar Observatory, currently under development at Caltech and JPL. It will use a 3368 active actuator deformable mirror to compensate the atmospheric wavefront on scales as fine as $8 \mathrm{~cm}$ at the telescope pupil, using both natural and sodium laser guidestars (NGS and LGS). Such fine correction provides unique new science opportunities, from extreme contrast on bright stars in the infrared to all-sky diffraction-limited imaging and spectroscopy in the visible. A suite of new back-end instruments is being developed to exploit of these capabilities, including the SWIFT visible-light integral field spectrograph, Project 1640, a near-infrared coronagraphic integral field spectrograph, and 888Cam, a high-resolution visible light imager.

Dekany et $\mathrm{al}^{2}{ }^{2}$ presented the science case for PALM-3000, which includes solar system, galactic, and extragalactic components. While ambitious, these science goals are limited, intended to complement the capabilities of present 8-10 $\mathrm{m}$ class telescopes equipped with AO. Out of the scientific considerations came the requirements for 1) Precision nearinfrared photometry and astrometry based on excellent point spread function stability and knowledge, 2) High contrast imaging at both visible and near-IR wavelengths, 3) High spatial resolution spectroscopy of resolved objects, and 4) High sensitivity faint-object visible-light imaging. All of these capabilities require only high-order, narrow field-of-view correction, subject to natural anisoplanatism. Each has some initial applicability based on NGS wavefront sensing, but all would also benefit greatly from high sky coverage fraction.

The preliminary design presented herein allows for a phased implementation of these science capabilities. During Phase 1 of the project, we will upgrade the NGS capabilities of the PALMAO system by installing a new deformable mirror, high-order wavefront sensor, and wavefront reconstructor computer. While primarily upgrading the NGS bright star performance, the current LGS capabilities will be retained with some modest improvement in Strehl. We expect to

* Send correspondence to A.H.B.: E-mail: abouchez@astro.caltech.edu, Telephone: 16263958915.

Adaptive Optics Systems, edited by Norbert Hubin, Claire E. Max, Peter L. Wizinowich, Proc. of SPIE Vol. 7015, 70150Z, (2008)

0277-786X/08/\$18 $\cdot$ doi: $10.1117 / 12.789777$

Proc. of SPIE Vol. 7015 70150Z-1 


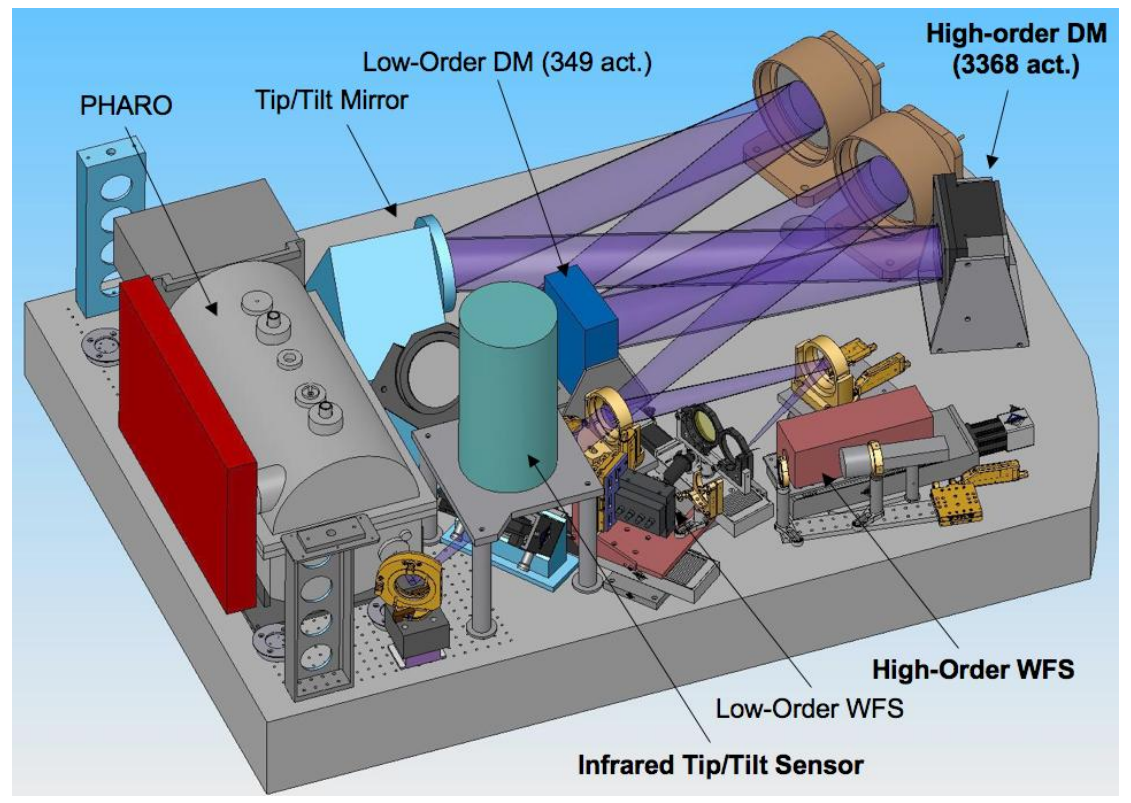

Figure 1. Conceptual model of the PALM-3000 optical bench, with new components labeled in bold.

commission the Phase 1 upgrade in 2010. Phase 2, which will consist of the addition of a $20 \mathrm{~W}$ class laser, an infrared tip/tilt sensor, and the replacement of the Truth Wavefront Sensor, will follow with installation planned in 2011.

\section{DESIGN}

The PALM-3000 system will be located at the Cassegrain focus of the Hale $5.1 \mathrm{~m}$ telescope. As an upgrade, PALM3000 will reuse many components of the present PALMAO system, including the optical bench, relay optics, internal stimulus, laser beam transfer and launch systems ${ }^{3}$, and handling fixtures. The critical new components will be a new high-order deformable mirror, a $64 \times 64$ subaperture high-order wavefront sensor, a powerful wavefront processor computer, and an infrared tip/tilt sensor. While the optical design of the AO relay has yet to be finalized, a preliminary model illustrating the location of the new components is shown in Figure 1.

\subsection{Deformable Mirrors}

High spatial frequency wavefront control will be provided by a Xinetics photonics module deformable mirror (DM) with 3368 active actuators on a $66 \times 66$ grid with $1.8 \mathrm{~mm}$ pitch (see Figure 2). As this "tweeter" DM has only $2 \mu \mathrm{m}$ of stroke, it must be supplemented by the current PALMAO DM, which has 349 actuators on a $7.0 \mathrm{~mm}$ pitch and $5 \mu \mathrm{m}$ of stroke. A single optical relay will re-image the Hale telescope pupil to an optical space between the woofer and tweeter DMs, with the DMs at identical incidence angle to keep their actuator patterns registered. A detailed optimization of DM conjugate height for corrected field of view and astrometric stability remains to be performed.

The 3368 actuator tweeter DM will be controlled by eight $4 \mathrm{U}$ electronics chassis developed by Xinetics, each providing 480 channels of 16 bit control over a $0-100 \mathrm{~V}$ range. A ninth chassis will control the 349 actuator woofer DM. We plan to locate the drive electronics remotely from the AO system in a climate-controlled computer room, to minimize power dissipation in the telescope dome.

\subsection{High Order Wavefront Sensor}

A high order Shack-Hartmann wavefront sensor ${ }^{4}$ will measure both NGS and LGS wavefronts on scales as fine as $8 \mathrm{~cm}$ across the Hale telescope pupil. The sensor uses an E2V CCD50 $128 \times 128$ pixel detector and SciMeasure camera and controller, which can be read out at 30 to $3000 \mathrm{~Hz}$ frame rate. Four selectable microlens arrays provide $8 \times 8,16 \times 16$, $32 \times 32$, and $64 \times 64$ subapertures, allowing the sensor to be adapted to a wide range of guidestar magnitudes. An adjustable spatial filter at input the focal plane will minimize aliasing in the fine pupil sampling modes ${ }^{5}$. 

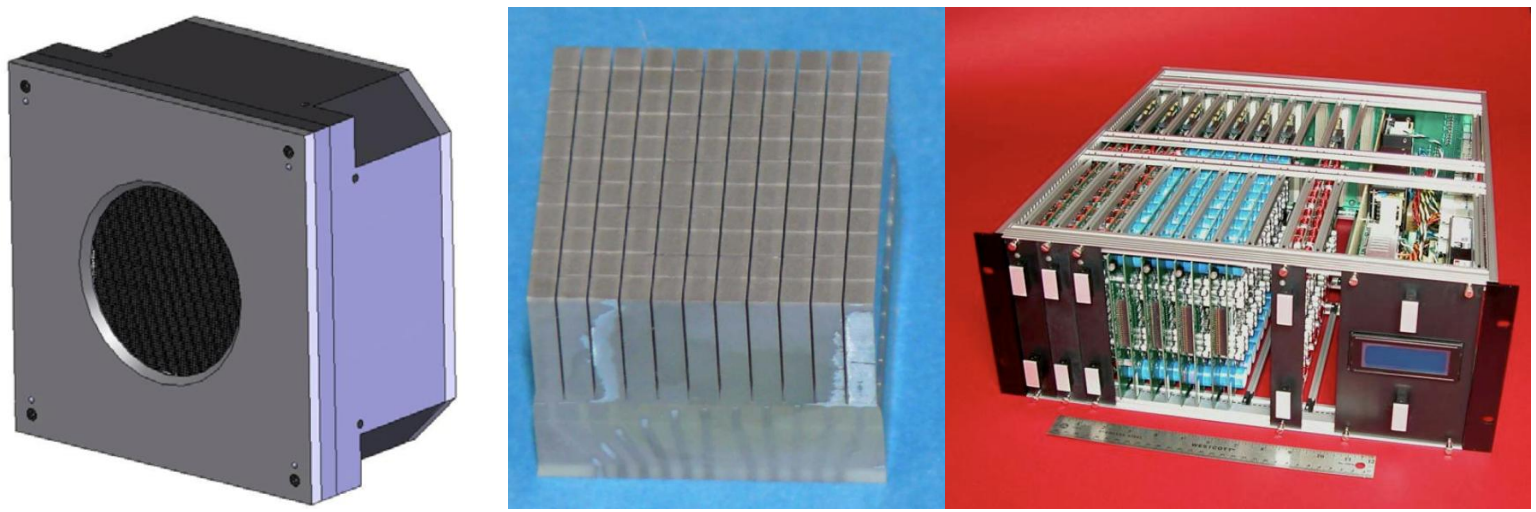

Figure 2. Left: Model of the 3368 active actuator deformable mirror. The mirror clear aperture is 4.55". Middle: Xinetics Phonics module, with $11 \times 11$ actuators machined into a single block of lead-magnesium-niobate ceramic. A $6 \times 6$ array of these modules is bonded to the mirror face sheet. Right: One drive electronics chassis, capable of controlling 480 actuators.

The very fine pupil sampling modes lead to a stringent requirement on DM to microlens pupil registration of $10 \%$ of the finest subaperture, or $0.16 \%$ of the pupil. This has driven us to a design using a tilted spherical mirror as a reflective collimator, which provides both freedom from longitudinal color, and anamorphic magnification to expand the foreshortened projection of the DM actuators on the microlens arrays, preserving the Fried geometry.

\subsection{Wavefront Processor Computer}

The PALM-3000 wavefront processor computer ${ }^{6}$ has been specified to allow full vector matrix multiplication reconstruction of the guidestar wavefront at $64 \times 64$ subapertures and $2 \mathrm{kHz}$ frame rate. Several candidate hardware architectures were evaluated for this challenging computational task, including fixed-point and floating-point digital signal processor boards, and a network of graphics processor units (GPUs). The selected system uses 16 NVIDIA model 8800 GPUs, hosted in a cluster of 8 desktop personal computers running the Linux operating system. Using entirely offthe-shelf hardware, this solution provides the greatest ease of use, potential upgradability, and cost savings. The computers are interconnected using a Quadrics QsNet switch with $14.4 \mathrm{~GB} / \mathrm{s}$ bandwidth, which also links them to a central node (with one GPU used for LGS low-order wavefront reconstruction) and a telemetry-recording PC. The entire network thus consists of 10 PCs housing 17 graphics cards.

By dividing the high-order wavefront reconstruction between 16 GPUs and further processing the HOWFS subapertures in two batches, starting when just half the pixels have been read, a total computational latency of just 222 $\mu$ s is achieved. We expect that this can be further reduced by future upgrades of the GPUs, which enjoy a rapid development cycle due to strong commercial demand.

The wavefront processor computer will be housed in two racks in a climate-controlled computer room, and will communicate with the wavefront sensors and deformable mirror drivers via fiber optics. All supervisory control and telemetry data extraction will be performed over standard Gigabit ethernet connections.

\subsection{Infrared Tip/Tilt Sensor}

The Phase 2 infrared tip/tilt sensor will be a direct imaging camera using a HAWAII2-RG detector with region-ofinterest readout capabilities, sensitive to the $\mathrm{J}$ and $\mathrm{H}$ bands. It will be housed in an end-looking cryostat whose axis is perpendicular to the optical bench. The detector pixels subtend $18 \mu \mathrm{m}$ at the native AO platescale, Nyquist sampling the diffraction-limited point-spread function in the $\mathrm{H}$ band. This makes possible an attractively simple optical design, with only an $85 \mathrm{~mm}$ diameter cryostat window $250 \mathrm{~mm}$ ahead of a cold infrared-blocking filter with cutoff at $1.78 \mu \mathrm{m}$ and detector. The solid angle subtended by the window is then small enough to increase the infrared sky background by $<40$ $\%$. The field of view of 135 arcseconds on the diagonal is sufficient to allow access to the required guide star patrol range without the need for field or sensor translation. 


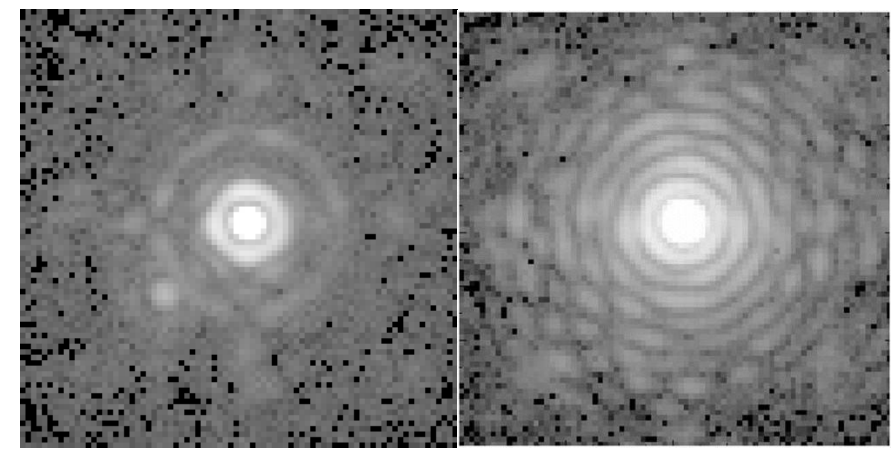

Figure 3. Left: $75 \%$ Strehl broad-band $2.14 \mu \mathrm{m}$ image of SAO 66571, recorded using the PALMAO system and PHARO, on 19 May 2008 in summer median conditions $\left(r_{0}=11 \mathrm{~cm}\right)$. This image exemplifies the present performance of the PALMAO system on NGS. The apparent companion in the lower left is an instrument artfact. Right: 94\% Strehl narrow-band $2.17 \mu \mathrm{m}$ image of HD 121107 obtained with the $1.5 \mathrm{~m}$ unobscured subaperture, PALMAO, and PHARO (from Ref. 7). While the diffraction pattern is broader, the Strehl is similar to that expected with PALM-3000 over the full $5.1 \mathrm{~m}$ telescope aperture. Both images are 2.0 " on a side.

The IRTT will be used in either an acquisition or tip/tilt control mode. In acquisition mode, a full-field integration will be read out and displayed to the user interface for guidestar selection. Upon selecting the guide star, a single quad cell of 4 pixels will be read out at up to $500 \mathrm{~Hz}$ to provide the tip/tilt control signal for LGS observing modes.

\section{PERFORMANCE}

Figure 3 (left) displays the high Strehl already achieved in the near-infrared by the PALMAO system. In median summer conditions $\left(\mathrm{r}_{0}=11 \mathrm{~cm}\right.$ ), the root-means squared (RMS) wavefront error using bright NGS is typically $\sim 190 \mathrm{~nm}$, allowing very stable imaging and precise photometry. The central goal of the PALM-3000 system is to further reduce this wavefront error by a factor of two, delivering over $90 \%$ Strehl in the K band on bright stars. This performance is illustrated in Figure 3 (right), recorded using the Well-Corrected Subaperture experiments by Serabyn et al. ${ }^{7}$, who reimaged an unobscured $1.5 \mathrm{~m}$ diameter subaperture onto the PALMAO deformable mirror, resulting in an interactuator spacing of $9.4 \mathrm{~cm}$. Using bright guide stars, they measured Strehl ratios of up to $94 \%$ at $2.17 \mu \mathrm{m}$ wavelength, similar to that which PALM-3000 will provide over the full $5.1 \mathrm{~m}$ aperture.

The predicted bright-star NGS performance of PALM-3000 is displayed in Figure 4 (left), for realistic observing conditions $\left(\mathrm{r}_{0}=9.2 \mathrm{~cm}\right.$ and $30^{\circ}$ zenith angle). Detailed error budget analysis (see Figure 5) predicts an RMS wavefront error of $94 \mathrm{~nm}$, providing extremely high performance in the near-infrared and diffraction-limited performance throughout the visible. Such low wavefront error makes possible both high contrast companion searches in the nearinfrared, and high sensitivity and stability imaging and spectroscopy in the visible and near-infrared, with spatial resolution as fine as 15 milliarcseconds at $360 \mathrm{~nm}$ wavelength.

A challenging LGS observing scenario is illustrated in Figure 4 (right), which displays the ensquared energy in $160 \times$ 160 mas spaxials when using an R=14.9 NGS 60" off-axis, equivalent to $5 \%$ sky coverage at galactic latitude $b=30^{\circ}$. While the PALMAO system provides very little light concentration shortward of $1.6 \mu \mathrm{m}$, PALM-3000 will enable high spatial resolution $0.65-1.00 \mu \mathrm{m}$ spectroscopy using SWIFT with great fidelity and sensitivity.

\section{INSTRUMENTS}

\subsection{PHARO}

The PHARO infrared camera and spectrograph ${ }^{8}$ is the current workhorse instrument of the PALMAO system. It provides imaging and grism spectroscopy with two image scales ( 25 and 40 miliarcserconds per pixel) in the $\mathrm{J}, \mathrm{H}$, and $\mathrm{K}$ bands $(1.16-2.36 \mu \mathrm{m})$, as well as a Lyot coronagraph. PHARO is routinely used as a platform for tests of novel coronagraphic masks ${ }^{7,9}$ and for observation with non-redundant pupil masking techniques ${ }^{10}$. With great flexibility and only $35 \mathrm{~nm}$ of internal wavefront error, PHARO is well suited to use with PALM-3000. To take full advantage of the improved wavefront of PALM-3000, we intend to add a Y band filter $(0.97-1.07 \mu \mathrm{m})$ to extend its use to shorter wavelengths. 

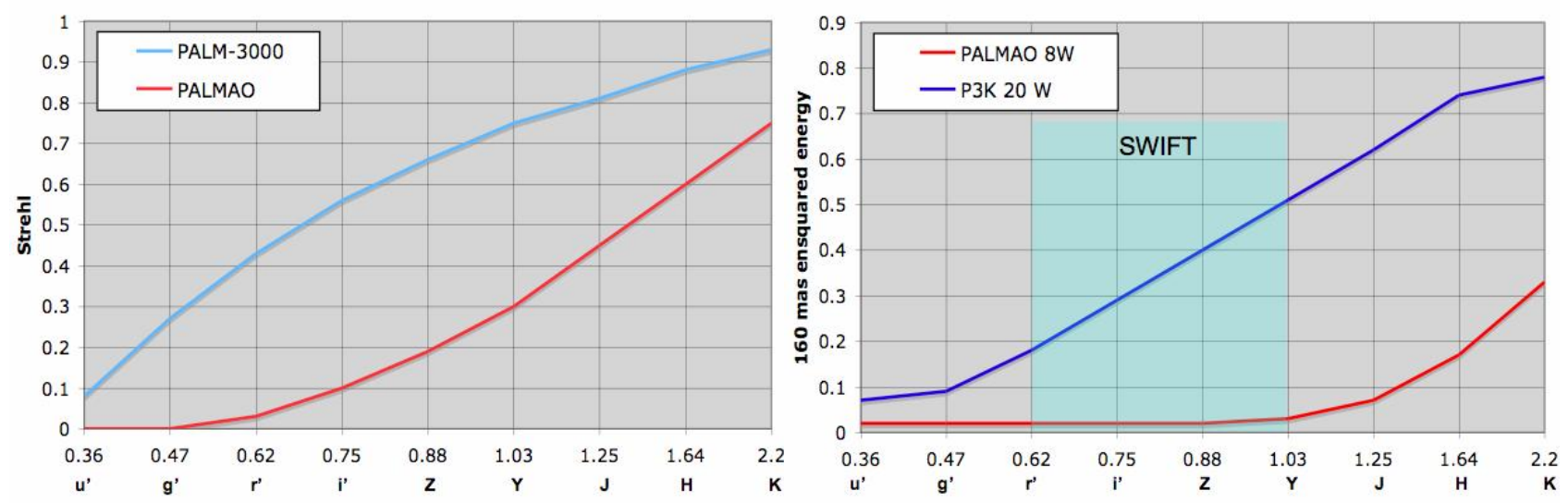

Figure 4. Left: Predicted Strehl as a function of observing band using a V=6 NGS, in median atmospheric conditions at $30^{\circ}$ zenith angle. Right: Predicted ensquared energy in a $160 \times 160$ mas spaxial using an R=14.9 guidestar 60" off-axis, for PALMAO with an 8 W micropulse-macropulse LGS and PALM-3000 with a $20 \mathrm{~W} \mathrm{CW-equivalent} \mathrm{LGS.} \mathrm{The} \mathrm{SWIFT} \mathrm{bandpass} \mathrm{is} \mathrm{indicated.}$

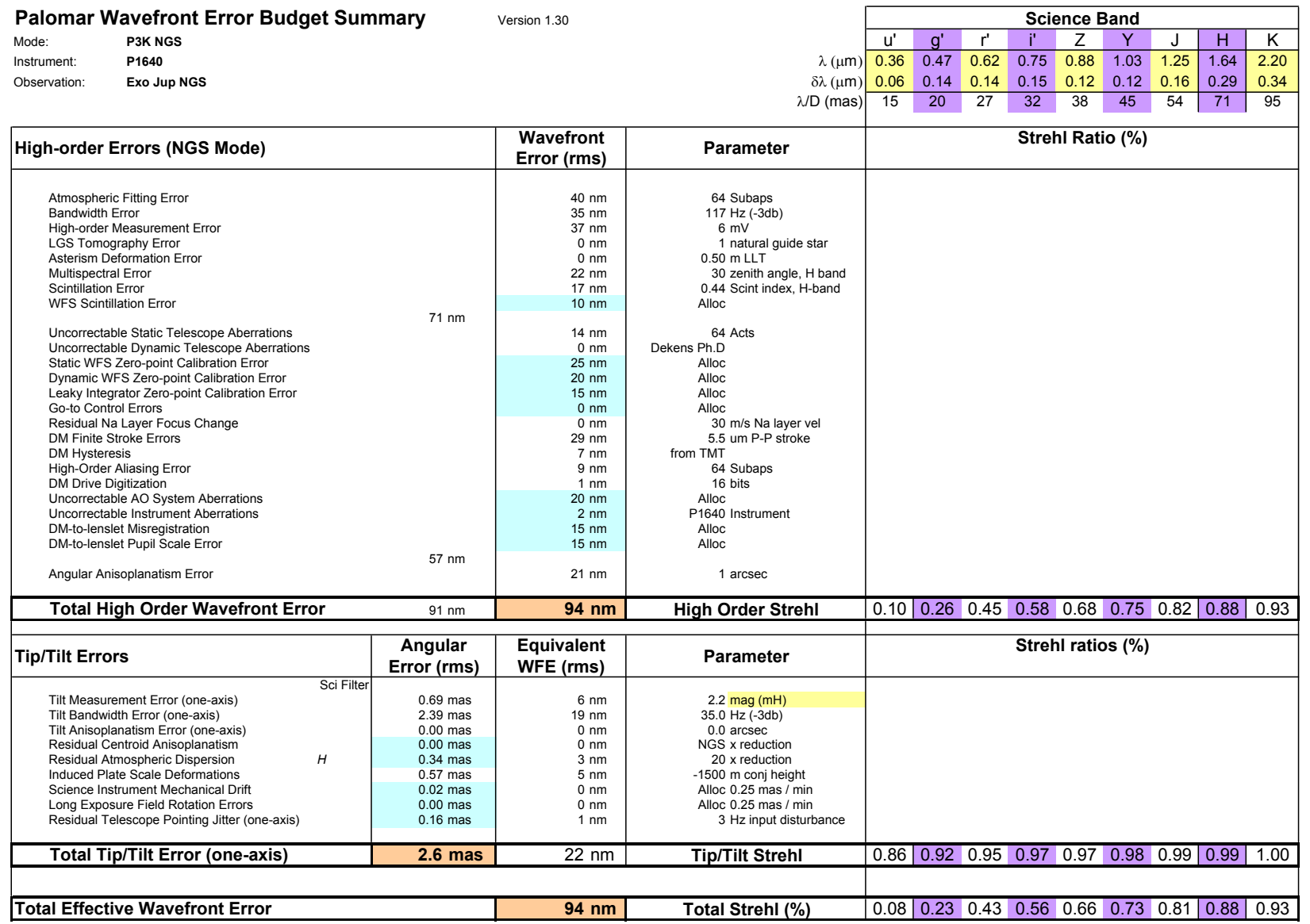

Figure 5. Detailed PALM-3000 error budget summary for one representative science case: High-contrast observations of nearby young stars $(\mathrm{V}=6)$ with the Project 1640 instrument. 


\subsection{Project 1640}

The Project 1640 instrument $^{11}$ (see Figure 6) will capitalize on the low wavefront error delivered by PALM-3000, implementing three stages of contrast enhancement to enable the detection of planetary companions to bright nearby stars. An apodized Lyot coronagraph first suppresses the on-axis starlight subsequent to AO correction. Next, a JPLbuilt calibration system using starlight intercepted by the coronagraphic stop will measure the slowly varying component of the residual wavefront error (the quasi-static speckles), and this information will be fed back into the AO control loop. Finally, the back-end instrument, a diffraction-limited, low-resolution $(\lambda / \Delta \lambda \sim 30)$ integral field spectrograph, enables candidate substellar and planetary companions to be distinguished from remnant speckles by their distinct chromatic signatures.

Project 1640 will be commissioned on the PALMAO system in July 2008, where it is expected to achieve a contrast level of $\sim 10^{-3}$ at 0.5 " separation from stars as faint as V=12. Following the PALM-3000 upgrade and the integration of the interferometric calibration system in 2010, the P1640 stellar coronagraph is predicted to achieve a stellar suppression of $<10^{-6}(\Delta \mathrm{H}>15$ magnitudes) at 0.5 " separation from a bright on-axis stellar source at $1.6 \mu \mathrm{m}$. This performance will enable a search for faint, low mass companions to nearby main sequence stars, young Jovian mass $\left(5 \mathrm{M}_{\text {Jup }}\right)$ companions to young stars, and imaging studies, including the possible addition of polarimetric measurements, of planet-forming disks.

\subsection{SWIFT}

$\mathrm{SWIFT}^{12}$ is an I-z band AO-assisted integral field spectrograph, designed to complement di raction-limited nearinfrared IFS on $8-10 \mathrm{~m}$ class telescopes in resolution and sensitivity by combining high order AO correction, low sky background, and sensitive deep depletion CCDs. SWIFT will have a fixed spectral resolution and format $(650-1050 \mathrm{~nm}$, R 4000) with selectable sampling scales of 0.24 ", 0.16 ”, 0.08 " (all with $44 \times 89$ spaxials). These three scales are intended to adapt the instrument to various levels of AO correction, from that of the current PALMAO system to PALM-3000 with an upgraded laser. SWIFT will be commissioned on the PALMAO system in late summer 2008.

\subsection{Cam}

The final instrument planned for first light of PALM-3000 is $888 \mathrm{Cam}$, a follow-on to LuckyCam ${ }^{13}$ which was deployed as a visitor instrument behind the PALMAO system in July 2007. 888Cam is a Lucky Imaging visible light camera based on a $1024 \times 1024$ Andor EMCCD detector with sensitivity from 350 to $1000 \mathrm{~nm}$. A pixel scale of 12 mas pixel ${ }^{-1}$ will nyquist sample the diffraction-limited PSF to $600 \mathrm{~nm}$ wavelength, at a frame rate of up to 50 frames per second (for a 5" $\times 5 "$ subarray).

\section{CONCLUSIONS}

With 3368 active actuators and an interactuator spacing in the pupil of just $8 \mathrm{~cm}$, PALM-3000 will be the first extreme AO system deployed for astronomy. It will deliver high Strehl in the near-infrared (over $85 \%$ in the $\mathrm{H}$ band) and diffraction-limited correction throughout the visible to a suite of instruments optimized for low wavefront error science. On bright natural guide stars, the Project 1640 coronagraphic IFU will achieve contrast levels of better than $10^{-6}$ at $0.5^{\prime \prime}$, making possible the direct imaging of Jovian planets around nearby young stars. With both the Gemini Planet Imager and the VLT SPHERE instrument being fielded in Chile, this high contrast capability is likely to remain unmatched in the northern hemisphere for some time. At shorter wavelengths, visible light integral field spectroscopy with SWIFT and diffraction-limited imaging with 888Cam will open a new wavelength realm for AO-corrected scientific observation on large telescopes, with sensitivity rivaling that of the Hubble Space Telescope.

\section{ACKNOWLEDGMENTS}

This research was supported by National Science Foundation grant AST-0619922, PI: Dr. R. Dekany. Support for the wavefront reconstructor computer has been provided by the Air Force Office of Sponsored Research, Award \#FA955006-1-0343 (DURIP FY06). Support for the PALM-3000 3368 actuator DM is provided by a NASA Small Business Innovation Research grant \#NNG06CA21C. Generous support for the adaptive optics interface to the SWIFT instrument has been provided by Ron and Glo Helin. 

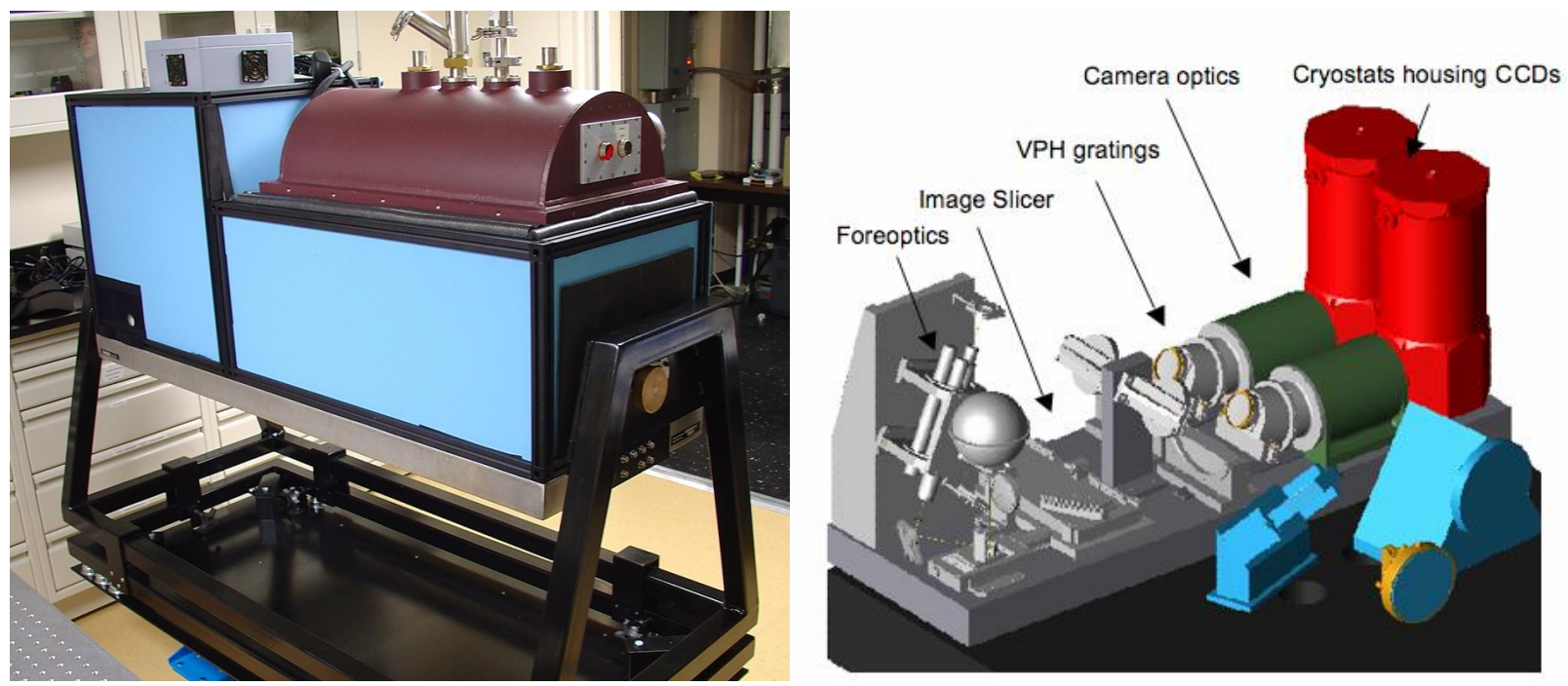

Figure 6. Left: Project 1640 instrument at the American Museum of Natural history, just prior to shipping to Palomar in June 2008. Right: Model of the SWIFT spectrograph mounted on the PALMAO bench, with major components labeled.

\section{REFERENCES}

1. M. Troy et al., "Palomar Adaptive Optics Project: Status and Performance", Proc. SPIE 4007, pp. 31-40, 2000.

2. R. G. Dekany et al., "PALM-3000: visible light AO on the 5.1 meter telescope", Proc. SPIE 6272, pp. 62720G, 2006.

3. J. Roberts et al., "Facilitizing the Palomar Laser Guidestar Adaptive Optics System", these proceedings.

4. C. Baranec, "High Order Wavefront Sensing System for PALM-3000", these proceedings.

5. L. Poyneer and B. Macintosh, "Spatially filtered wave-front sensor for high-order adaptive optics," J. Opt. Soc. Am. A. 21, pp. 810-819, 2004.

6. T. Truong et al., "Real-time Wavefront Control for the PALM-3000 High Order Adaptive Optics System", these proceedings.

7. E. Serabyn, K. Wallace, M. Troy, B. Mennesson, P. Haguenauer, R. Gappinger, and R. Burruss. "Extreme Adaptive Optics Imaging with a Clear and Well-Corrected Off-Axis Telescope Subaperture". AJ 658, pp. 13861391, 2007.

8. T. L. Hayward, B. Brandl, B. Pirger, C. Blacken, G. E. Gull, J. Shoenwald, and J. R. Houck, "PHARO: A NearInfrared Camera for the Palomar Adaptive Optics System," PASP 113, pp. 105-118, 2001.

9. J. R. Crepp, E. Serabyn, J. Carson, J. Ge, I. Kravchenko, R. Burruss, and K. Wallace, "A Direct Imaging Search for Circumbinary Brown Dwarfs", AAS Meeting \#211, \#30.01, 2007.

10. F. Martinache, J. P. Lloyd, M. J. Ireland, R. S. Yamada, and P. G. Tuthill, "Precision Masses of the Low-Mass Binary System GJ 623", $A J$ 661, pp. 496-501, 2007.

11. S. Hinkley, B. R. Oppenheimer, D. S. Brenner, A. Sivaramakrishnan, R. Soummer, I. R. Parry, and D. L. King. "A new integral field spectrograph for exoplanetary science at Palomar", these proceedings.

12. N. Thatte, M. Tecza, F. Clarke, T. Goodsall, J. Lynn, D. Freeman, and R. L. Davies, "The Oxford SWIFT Integral Field Spectrograph", Proc. SPIE 6269, pp. 62693L, 2006.

13. N. M. Law, C. D. Mackay, R. G. Dekany, M. Ireland, J. P. Lloyd, A. M. Moore, P. Tuthill, and H. Woodruff, "Getting Lucky with Adaptive Optics: Fast AO Image Selection in the Visible with a Large Telescope," Submitted to ApJ 805, 2008. 